\title{
THE CITATION IMPACT OF DIGITAL PREPRINT ARCHIVES FOR SOLAR PHYSICS PAPERS*
}

\author{
TRAVIS S. METCALFE \\ High Altitude Observatory and Scientific Computing Division, NCAR, \\ PO Box 3000, Boulder, CO 80307, U.S.A. \\ (e-mail: travis@ucar.edu)
}

(Received 5 July 2006; accepted 16 October 2006; Published online 30 November 2006)

\begin{abstract}
Papers that are posted to a digital preprint archive are typically cited twice as often as papers that are not posted. This has been demonstrated for papers published in a wide variety of journals, and in many different subfields of astronomy. Most astronomers now use the arXiv.org server (astro-ph) to distribute preprints, but the solar physics community has an independent archive hosted at Montana State University. For several samples of solar physics papers published in 2003, I quantify the boost in citation rates for preprints posted to each of these servers. I show that papers on the MSU archive typically have citation rates 1.7 times higher than the average of similar papers that are not posted as preprints, while those posted to astro-ph get 2.6 times the average. A comparable boost is found for papers published in conference proceedings, suggesting that the higher citation rates are not the result of self-selection of above-average papers.
\end{abstract}

\section{Background}

The arXiv.org preprint server $\left(\right.$ astro-ph $\left.h^{1}\right)$ has been operating since 1992 (Ginsparg, 2001 ) and now includes about $80 \%$ of all new papers published in major refereed astrophysics journals around the globe (Metcalfe, 2005). Because it is the single source that many astronomers now use to keep up with the literature, papers that are posted to astro-ph are cited roughly twice as often as papers that are not posted. This has been shown to have little to do with the significance of the paper - even conference proceedings are cited twice as often when posted, although still 20 times less overall (Schwarz and Kennicutt, 2004).

Falling somewhere between earth sciences and astrophysics, the solar physics community has been slower to adopt preprint archives than some subfields, with only about $7 \%$ of Solar Physics papers posted to astro-ph in 2005, compared to about $79 \%$ of papers published in Astrophysical Journal, and less than $1 \%$ of papers published in Geophysical Research Letters. An independent solar physics archive was established in 1999 at Montana State University $\left(\mathrm{MSU}^{2}\right)$, which has

*Editors' Note: This paper lies outside the normal purview of Solar Physics papers, however the editors feel that the content is of sufficient importance for all Solar Physics authors and readers to merit its publication.

${ }^{1}$ http://arXiv.org/archive/astro-ph.

${ }^{2}$ http://solar.physics.montana.edu/cgi-bin/eprint/index.pl. 
attracted a slightly larger following with $17 \%$ of Solar Physics papers posted last year. The adoption rate is slightly higher among solar physicists who publish in broader astrophysics journals, with about 19\% of Astronomy and Astrophysics papers and 29\% of Astrophysical Journal papers in this subfield posted to astro-ph in 2005.

With so much potential for growth in the adoption rate of preprint archives among the solar physics community, several questions arise: (1) Do papers in this subfield enjoy a similar boost in citation rates when posted to one of these archives?

(2) Is there evidence that the boost is not due to self-selection of better papers, and (3) Is there any quantitative reason to prefer one of the archives over the other? This paper aims to answer these questions with citation statistics obtained using NASA's Astrophysics Data System (Kurtz et al., 2000) for three samples of papers published in 2003. This is far enough in the past to generate significant citation counts, while recent enough to sample a higher adoption rate for the preprint archives.

\section{Methodology and Results}

Although the citation database of the Astrophysics Data System (ADS) is not complete, it includes data from all of the major astronomy and physics journals since 1999. Any incompleteness should have a uniform effect on all of the papers considered in this section.

\subsection{CAN PREPRINT ARCHIVES BOOST CITATION RATES?}

Our first sample includes the 171 papers published in Solar Physics during 2003, which have collectively received 512 citations that are included in the ADS database (as of June 15, 2006). To isolate the citation impact of the individual preprint archives, we exclude one paper that was posted to both, which received a total of six citations. In 2003, just seven Solar Physics papers were posted exclusively to the MSU archive (receiving 33 citations), while six were posted only to astro-ph (43 citations) - leaving 157 unposted papers to account for the remaining 430 citations. Despite the small numbers, there is a significant difference in the average citation rates of these three sets of papers (see Table I, where errors are assigned from $\sqrt{N}$ uncertainties on the citation counts). Compared to the sample of unposted papers, preprints posted to the MSU archive received an average of $1.7 \pm 0.3$ times as many citations, while those posted to astro-ph received an average of $2.6 \pm 0.4$ times as many. Schwarz and Kennicutt (2004, Figure 9) found no significant difference in the long-term citation patterns of papers posted before and after peer review, so this citation boost is not merely due to the slightly earlier availability of the paper. 
TABLE I

The citation impact of digital preprint archives for solar physics papers.

\begin{tabular}{|c|c|c|c|c|c|c|}
\hline \multirow[b]{2}{*}{ Sample } & \multicolumn{2}{|c|}{ Unposted } & \multicolumn{2}{|c|}{ MSU archive } & \multicolumn{2}{|r|}{ astro-ph } \\
\hline & $\#$ & $\langle$ citations $\rangle$ & $\#$ & $\langle$ citations $\rangle$ & \# & $\langle$ citations〉 \\
\hline Solar Physics & 157 & $2.7 \pm 0.1$ & 7 & $4.7 \pm 0.8$ & 6 & $7.2 \pm 1.1$ \\
\hline IAU Symp. 210 & 150 & $0.24 \pm 0.04$ & 0 & $\ldots$ & 20 & $0.65 \pm 0.18$ \\
\hline MSU archive & 0 & $\ldots$ & 82 & $7.6 \pm 0.3$ & 20 & $10.2 \pm 0.7$ \\
\hline
\end{tabular}

\subsection{ARE THE CITATION RATES HIGHER FROM SELF-SELECTION?}

Confronted with the data in Table I, many scientists will wonder whether there is a self-selection effect. Perhaps the papers on the preprint archives are not representative of the full sample, and the authors chose to post them because they were better than average? This could explain why these papers received more citations.

We can address this question by examining a second sample of papers that we would not expect authors to self-select for high quality. Schwarz and Kennicutt (2004) found that conference proceedings are typically cited about 20 times less than refereed journal papers, so we choose a solar physics proceedings published in 2003, from IAU Symposium 210 "Modelling of Stellar Atmospheres." Of the 170 papers included in this volume, only 20 were posted to astro-ph, and none were posted to the MSU archive. As expected, the overall rate of citations is much lower - with 13 citations to the 20 posted papers, and 36 citations to the 150 unposted papers (see Table I). Even conference proceedings papers, though cited an order of magnitude less overall, are cited $2.7 \pm 0.9$ times more when posted to astro$p h$. This is consistent with the factor of $2.6 \pm 0.4$ we found for Solar Physics papers.

\subsection{WHICH PREPRINT ARCHIVE SHOULD SOLAR PHYSICISTS USE?}

It is clear that both preprint archives provide a significant boost in the citation rates of posted papers, but the evidence suggests that astro-ph provides a slightly larger boost than the MSU archive. We can perform an additional test of the relative impact of the two archives by looking at a third sample of papers. There were 102 refereed papers posted to the MSU archive in 2003, and 20 were also posted to astro-ph. The papers posted to both preprint archives received 204 citations, while the 82 papers posted only to the MSU archive garnered $621 \mathrm{ci}$ tations (see Table I). This implies an additional boost of $1.3 \pm 0.1$ for papers posted to astro-ph, on top of the factor of approximately two boost from posting to the MSU archive. This is roughly consistent with the citation impact of astro-ph alone. 
Note that the average citation rate for all refereed papers on the MSU archive (most of them published in broader astrophysics journals ${ }^{3}$ ) is systematically higher than that for Solar Physics papers alone. In fact, astrophysics papers posted to the MSU archive have citation rates comparable to Solar Physics papers posted to astro-ph. This implies that the higher citation rates are connected to awareness of the paper among the broader astrophysics community either by publication in an astrophysics journal, or through a preprint posted to astro-ph. The highest average citation rates occur for papers that have been posted to both preprint archives, though astro-ph appears to be the best single choice.

\section{Conclusions and Discussion}

Despite the slower adoption by the solar physics community, digital preprint archives boost the citation rates of posted papers to twice the level of unposted papers, a conclusion first noted in the comprehensive study of Schwarz and Kennicutt (2004). The evidence suggests that, like many other subfields in astronomy, the citation rate is elevated from the improved visibility of the paper rather than from self-selection by authors choosing to post above-average papers. Unlike other subfields, solar physicists maintain an independent preprint archive which also boosts citation rates, though the broader user-base of astro-ph provides a larger boost.

If citation rates track the assimilation of new results by the community, then astro-ph seems to be the best single form of communication available. Editors who want to maximize the impact of their journals should encourage authors to post their preprints to astro-ph. Authors in solar physics, where astro-ph is currently underutilized, should consider the advantages that other subfields have already discovered.

\section{Acknowledgements}

I would like to thank Sarah Gibson for a comment that helped motivate this work, and Stan Solomon for several constructive suggestions. This research made use of NASA's ADS. The National Center for Atmospheric Research is a federally funded research and development center sponsored by the National Science Foundation.

\footnotetext{
${ }^{3}$ Among papers in the MSU sample, $41 \%$ were published in Astrophysical Journal, $25 \%$ in Astronomy and Astrophysics, and $11 \%$ in Solar Physics. There was no significant difference in the adoption rate of astro-ph for these three subsamples.
} 


\section{References}

Ginsparg, P.: 2001, in D. Shaw (ed.), Electronic Publishing in Science, UNESCO, Paris, p. 41.

Kurtz, M.J., Eichhorn, G., Accomazzi, A., Grant, C.S., Murray, S.S., and Watson, J.M.: 2000, Astron. Astrophys. Suppl. Ser. 143, 41.

Metcalfe, T.S.: 2005, Bull. Am. Astron. Soc. 37, 555 (astro-ph/0503519).

Schwarz, G.J. and Kennicutt, R.C., Jr.: 2004, Bull. Am. Astron. Soc. 36, 1654 (astro-ph/0411275). 